COMMENT

https://doi.org/10.1038/s41467-019-13684-3

\title{
OPEN
}

\section{Limited antibody specificity compromises epitranscriptomic analyses}

\author{
Mark Helm (iD ${ }^{1 \star}$, Frank Lyko (1) ${ }^{2 \star} \&$ Yuri Motorin ${ }^{3 \star}$
}

A controversial discussion on the occurrence of the RNA modification $m^{1} A$ in mRNA takes a new turn, as an antibody with a central role in modification mapping was shown to also bind mRNA cap structures.

\section{Antibodies as tools for modification mapping}

RNA modifications are chemical alterations that diversify the functionality of the canonical RNA building blocks. New mapping methods have focused considerable attention on the modification content of eukaryotic mRNA and its potential for the regulation of gene expression.

A recent study reports on the differential specificity of antibodies directed against the RNA modification $\mathrm{m}^{1} \mathrm{~A}$, and how they impact interpretation of the resulting modification maps ${ }^{1}$. Particularly striking is the revelation that a previously used antibody binds cap structures in addition to $\mathrm{m}^{1} \mathrm{~A}$, and that previously reported $\mathrm{m}^{1} \mathrm{~A}$ mapping results likely contain an abundance of false positives at the $5^{\prime}$-end of mRNA.

While variabilities in antibody specificity are commonplace, the study by Jaffrey and colleagues will perhaps drive home the importance of specificity validation for antibody-dependent RNA modification mapping. What makes this case so relevant? Antibody biotechnology-and the many important tools it has produced-often rely on a combinatorial approach to identifying molecules with high affinity to specific epitopes. In the context of peptide binding, the variable region of typical antibodies recognizes an epitope of 5-12 amino ccids $^{2}$, which present a significant diversity of functional groups to mediate affinity and specificity. This situation is different for nucleic acids, as they possess comparatively limited structural diversity, and correspondingly less well-defined primary epitopes.

Antibodies directed against nucleic acid modifications have played an important role in the fields of epigenetics and epitranscriptomics. Enrichment of DNA containing $5 \mathrm{mC}$ by methylated DNA immunoprecipiatation (MeDIP) has been a widely used technique in epigenetics for decades, before its RNA version, MeRIP became popular. Indeed, MeRIP experiments have been published as early as the $1980 \mathrm{~s}^{3}$, albeit not under that acronym. Only the combination with RNA-Seq transformed it into a breakthrough technology for the RNA modification field in 2012, when two teams independently reported maps of $\mathrm{m}^{6} \mathrm{~A}$ modifications in mammalian $\mathrm{mRNA}^{4,5}$. Since then, several antibodies have been used for mapping various RNA modifications ${ }^{6}$ with substantial impact for the community. Problems with antibody specificity have been discussed ${ }^{7}$, but have remained largely under-recognized.

\footnotetext{
${ }^{1}$ Institute of Pharmacy and Biochemistry, Johannes Gutenberg-University Mainz, Staudingerweg 5, 55128 Mainz, Germany. ${ }^{2}$ Division of Epigenetics, DKFZZMBH Alliance, German Cancer Research Center, Heidelberg, Germany. ${ }^{3}$ IMoPA, UMR7365 CNRS-University of Lorraine, Biopôle, 9 Avenue de la Forêt de Haye, 54505 Vandœuvre-lès-Nancy, France. *email: mhelm@uni-mainz.de; f.lyko@dkfz-heidelberg.de; motorine5@univ-lorraine.fr
} 


\section{Off-target binding of antibodies is a widespread problem}

More recently, studies in the DNA modification field have begun to identify sources of artifacts. For example, issues of inherent cross-reactivity can be amplified by low abundance of the primary epitope. This is exemplified by the cross-reactivity of antibodies to contaminating bacterial nucleic acids that can confound the modification analysis of eukaryotic DNA ${ }^{8}$. Of note, the existence of $\mathrm{dm}^{6} \mathrm{~A}$ and $\mathrm{dm}^{4} \mathrm{C}$ in DNA of higher eukaryotes ${ }^{9,10}$ has been questioned by an antibody-independent analysis ${ }^{11}$. Another recent study has shown that several antibodies directed toward DNA modification cross-react with short tandem repeats in a modification-independent manner, which can in turn generate experimental noise as high as $99 \% 12$.

Given that the development of such antibodies includes a conjugation step to a protein via the oxidized sugar moiety of a modified nucleoside ${ }^{13}$, modification-specific antibodies could be expected to recognize the modified nucleobase irrespective of whether they are found in DNA or RNA. Thus, the demonstration of specificity problems in MeRIP experiments in the current publication by Grozhik et al. should not come as a surprise; rather it is a long awaited, experimentally thorough and convincing demonstration of antibody-dependent artifacts in the RNA modification field ${ }^{1}$. In addition to providing experimental guidelines for the field as a whole, the study also uncovers the unexpected binding of a commercially available anti- $\mathrm{m}^{1} \mathrm{~A}$ antibody to cap structures. Furthermore, the study provides important clarifications in the controversial discussion regarding the number of $\mathrm{m}^{1} \mathrm{~A}$ residues present in mammalian mRNA, which have been reported in several publications ${ }^{14-17}$. More specifically, the results reported by Grozhik et al. suggest that $\mathrm{m}^{1} \mathrm{~A}$ is infrequent in mRNA, and that the prevalence of this modification was substantially overestimated in previous studies. A comparative assessment of two $\mathrm{m}^{1} \mathrm{~A}$ antibodies led to vastly different results in MeRIP-type experiments, likely pointing to a general problem in the field. For one, specifications and specificity claims for a given antibody should be taken with caution and preferably confirmed for each application using the relevant controls. Secondly, it should now be clear that confirming antibody specificity by simple methods such as dot blot experiments should be considered insufficient ${ }^{18}$. Of the many validation techniques that the field has developed ${ }^{6}$, Grozhik et al. judiciously applied mass spectrometry and thin layer chromatography to characterize the physicochemical properties of material isolated by MeRIP ${ }^{1}$. A systematic characterization of the various antibodies commonly used might be highly beneficial, as was shown in the not-so-distant field of histone modifications. There, a systematic evaluation of antibody specificity was conducted using peptide-arrays, and revealed substantial specificity problems already several years ago ${ }^{19}$.

\section{Considerations beyond antibody specificity}

On a more fundamental level, one might question if a single methyl group in a nucleic acid fragment can really provide a sufficient level of selectivity for MeRIP or other similar techniques. Although our understanding of binding modes is limited, it is clear that the primary epitope can not only be the modification itself (i.e. a methyl group), but can extend to the modified nucleobase (i.e. adenine) and beyond. It follows that all adenines present in the RNA also compete for binding, albeit with lower affinity than the methylated adenine. In such a situation, the enrichment will be governed by the relative affinities toward modified and unmodified residues, and by their relative abundances. This, in turn, means, that any adenine in unmodified RNA (including polyA-tails) may give rise to non-specific binding, especially if the modification is of low abundance. It is thus perhaps not surprising that enrichment factors reported in MeRIP experiments are as low as $4-10-$ fold $^{20}$. For relatively abundant modifications, such as $\mathrm{m}^{6} \mathrm{~A}$, this may still be sufficient to produce credible mapping results. However, this may not be the case for less abundant RNA modifications.

With respect to MeRIP in general, several additional problems exist that extend beyond antibody specificity, and which could skew the results of modification mapping experiments. A number of these problems are related to the experimental design of Illumina sequencing and library preparation protocols used. For example, early modification calling reports have neglected the use of unique molecular identifiers $(\mathrm{UMI})^{6}$, leading to artificial amplification of noise by $\mathrm{PCR}^{21}$. Potential artifacts resulting from RNA-Seq adapter design have also been discussed $^{22}$. Moreover, problems in the computational analysis of RNA-Seq data, such as ambiguity in read mapping, are among the known error sources ${ }^{23}$. Finally, the field needs standards for stringent statistical data analysis ${ }^{24}$. Taking into account all these limitations, the massive datasets obtained by newly reported mapping techniques for RNA modification analysis should be considered collections of candidate modification sites, rather than experimentally confirmed modification landscapes. Therefore, further efforts should aim to develop and apply multiple orthogonal methods for the validation of modification sites and genome-wide patterns ${ }^{6}$.

Received: 9 July 2019; Accepted: 14 November 2019; Published online: 11 December 2019

\section{References}

1. Grozhik, A. V. et al. Antibody cross-reactivity accounts for widespread appearance of m1A in 5'UTRs. Nat. Commun. 10, 5126 (2019).

2. Stave, J. W. \& Lindpaintner, K. Antibody and antigen contact residues define epitope and paratope size and structure. J. Immunol. 191, 1428-1435 (2013).

3. Horowitz, S., Horowitz, A., Nilsen, T. W., Munns, T. W. \& Rottman, F. M. Mapping of N6-methyladenosine residues in bovine prolactin mRNA. Proc. Natl Acad. Sci. USA 81, 5667-5671 (1984).

4. Meyer, K. D. et al. Comprehensive analysis of mRNA methylation reveals enrichment in 3' UTRs and near stop codons. Cell 149, 1635-1646 (2012)

5. Dominissini, D. et al. Topology of the human and mouse m6A RNA methylomes revealed by m6A-seq. Nature 485, 201-206 (2012).

6. Helm, M. \& Motorin, Y. Detecting RNA modifications in the epitranscriptome: predict and validate. Nat. Rev. Genet. 18, 275-291 (2017).

7. Mishima, E. et al. Immuno-northern blotting: detection of RNA modifications by using antibodies against modified nucleosides. PLoS ONE 10, e0143756 (2015).

8. O'Brown, Z. K. et al. Sources of artifact in measurements of $6 \mathrm{~mA}$ and $4 \mathrm{mC}$ abundance in eukaryotic genomic DNA. BMC Genomics 20, 445 (2019).

9. Liu, J. et al. Abundant DNA 6mA methylation during early embryogenesis of zebrafish and pig. Nat. Commun. 7, 13052 (2016).

10. Zhang, G. et al. N6-methyladenine DNA modification in Drosophila. Cell 161, 893-906 (2015).

11. Schiffers, S. et al. Quantitative LC-MS provides no evidence for $\mathrm{m}(6) \mathrm{dA}$ or $\mathrm{m}$ (4) dC in the genome of mouse embryonic stem cells and tissues. Angew. Chem. Int. Ed. Engl. 56, 11268-11271 (2017).

12. Lentini, A. et al. A reassessment of DNA-immunoprecipitation-based genomic profiling. Nat. Methods 15, 499-504 (2018).

13. Feederle, R. \& Schepers, A. Antibodies specific for nucleic acid modifications. RNA Biol. 14, 1089-1098 (2017).

14. Li, X. et al. Base-resolution mapping reveals distinct $\mathrm{m}(1) \mathrm{A}$ methylome in nuclear- and mitochondrial-encoded transcripts. Mol. Cell 68, 993-1005.e9 (2017).

15. Schwartz, S. m(1)A within cytoplasmic mRNAs at single nucleotide resolution: a reconciled transcriptome-wide map. RNA 24, 1427-1436 (2018).

16. Dominissini, D. et al. The dynamic N(1)-methyladenosine methylome in eukaryotic messenger RNA. Nature 530, 441-446 (2016).

17. Safra, M. et al. The m1A landscape on cytosolic and mitochondrial mRNA at single-base resolution. Nature 551, 251-255 (2017). 
18. Delatte, B. et al. RNA biochemistry. Transcriptome-wide distribution and function of RNA hydroxymethylcytosine. Science 351, 282-285 (2016).

19. Rothbart, S. B. et al. An interactive database for the assessment of histone antibody specificity. Mol. Cell 59, 502-511 (2015).

20. Slama, K. et al. Determination of enrichment factors for modified RNA in MeRIP experiments. Methods 156, 102-109 (2019).

21. Squires, J. E. et al. Widespread occurrence of 5-methylcytosine in human coding and non-coding RNA. Nucleic Acids Res. 40, 5023-5033 (2012).

22. Dai, Q. et al. Nm-seq maps 2'-O-methylation sites in human mRNA with base precision. Nat. Methods 14, 695-698 (2017).

23. Schwartz, S. \& Motorin, Y. Next-generation sequencing technologies for detection of modified nucleotides in RNAs. RNA Biol. 14, 1124-1137 (2017).

24. Legrand, C. et al. Statistically robust methylation calling for wholetranscriptome bisulfite sequencing reveals distinct methylation patterns for mouse RNAs. Genome Res. 27, 1589-1596 (2017).

\section{Author contributions}

M.H., F.L. and Y.M. conceived and wrote the manuscript.

\section{Competing interests}

The authors declare no competing interests.

\section{Additional information}

Correspondence and requests for materials should be addressed to M.H., F.L. or Y.M.

Reprints and permission information is available at http://www.nature.com/reprints

Publisher's note Springer Nature remains neutral with regard to jurisdictional claims in published maps and institutional affiliations.

(c) (i) Open Access This article is licensed under a Creative Commons Attribution 4.0 International License, which permits use, sharing, adaptation, distribution and reproduction in any medium or format, as long as you give appropriate credit to the original author(s) and the source, provide a link to the Creative Commons license, and indicate if changes were made. The images or other third party material in this article are included in the article's Creative Commons license, unless indicated otherwise in a credit line to the material. If material is not included in the article's Creative Commons license and your intended use is not permitted by statutory regulation or exceeds the permitted use, you will need to obtain permission directly from the copyright holder. To view a copy of this license, visit http://creativecommons.org/ licenses/by/4.0/.

(C) The Author(s) 2019 